\title{
Cooperação em saúde na perspectiva bioética
}

\author{
Cooperation in health from the bioethical perspective
}

José Paranaguá de Santana ${ }^{1}$

Volnei Garrafa ${ }^{2}$

${ }^{1}$ Núcleo de Estudos sobre Bioética e Diplomacia em Saúde, Opas/Unb/Fiocruz. Campus Darcy Ribeiro, L3 Norte, Gleba A, SC 4, Sala 206. 70.910-900 Brasília DF.paranagua@fiocruz.br ${ }^{2}$ Programa de

Pós-Graduação em Bioética, Cátedra UNESCO de Bioética/Núcleo de Estudos e Pesquisas em Bioética, Universidade de Brasília.

\begin{abstract}
This study considers the scenario of international relations in the transition to the twenty-first century as a backdrop for reflection on the bioethical perspective of international cooperation in health. It presents an exploratory analysis of the interdisciplinary scientific production in bioethics and public health in the international context, revealing that the focus and confluence of both issues has scant coverage in terms of diplomatic relations. It describes the methodology used to select publications cataloged in this interdisciplinary area from two bibliographic sources available on the web (93 articles in BVS/BIREME and 161 in PubMed), pointing to difficulties in locating this literature. The potential of the epistemological approach that flourished in Latin America under the guise of the Bioethics of Intervention in addressing the challenges that confront the international cooperation system, identified as the benchmark for anal$y$ sis of South-South cooperation in health, is recommended. It concludes by proposing systematization and broadening of knowledge at the intersection of bioethics, public health and diplomacy, whose projection in the political and institutional field can contribute to reducing inequalities in health conditions among nations.
\end{abstract}

Key words Bioethics, Health diplomacy, International health, Technical cooperation, International cooperation
Resumo O estudo considera o cenário das relações internacionais na transição para o Século XXI como pano de fundo para uma reflexão sobre a perspectiva bioética da cooperação internacional em saúde. Apresenta uma análise exploratória sobre a produção científica interdisciplinar da bioética com a saúde pública no contexto internacional, revelando que o enfoque de ambas, ou mesmo das articulações entre esses dois temas, tem parca abordagem do ponto de vista das relações diplomáticas. Descreve a metodologia que permitiu selecionar publicações catalogadas nessa área interdisciplinar em duas fontes bibliográficas disponiveis na Web (93 artigos na BVS/Bireme e 161 na Pub$\mathrm{Med}$ ), apontando dificuldades na recuperação dessa literatura. Advoga o potencial da vertente epistemológica que floresceu na América Latina sob a designação da Bioética de Intervenção na abordagem dos desafios que afrontam o sistema de cooperação internacional, acenado como referencial de análise da cooperação sul-sul em saúde. Conclui propondo a sistematização e o aprofundamento do conhecimento na interseção da bioética com a saúde pública e a diplomacia, cuja projeção no âmbito político-institucional poderá contribuir para a redução das desigualdades das condições de saúde entre as nações.

Palavras-chave Bioética, Diplomacia em saúde, Saúde internacional, Cooperação técnica, Cooperação internacional 


\section{Introdução}

A cooperação técnica entre países pressupõe a sintonia das relações internacionais orientada pela solidariedade, consenso e equidade ${ }^{1}$. No entanto, a aplicação desses postulados é modulada numa teia de interesses que resultam em condicionalidades na formulação e execução de projetos, cujos efeitos nem sempre conduzem à superação das formas tradicionais de colonialismo travestidas de apoio externo ao desenvolvimento econômico, científico e tecnológico dos países periféricos $^{2}$.

Durante pelo menos metade do século passado as relações internacionais alinhavaram-se em torno de dois eixos geopolíticos: o leste-oeste, de orientação ideológica separando os países alinhados ao capitalismo sob liderança dos Estados Unidos versus o bloco socialista sob a égide da União Soviética; e o norte-sul, fundamentado em critérios de estágio de desenvolvimento ou poder econômico, associado a características históricas, culturais e ambientais dos países situados de cada lado de um "[...] abismo em matéria de riqueza e saúde que separa ricos e pobres" ${ }^{3}$. No contexto de desigualdade e dependência nas relações políticas, econômicas e militares nesse mundo bipo$\operatorname{lar}^{4}$, dificilmente poderiam vicejar propostas genuínas de cooperação horizontal com resultados vantajosos para os países mais pobres.

Na transição para o século XXI configurouse um novo cenário que desafia os estudiosos da formação e das transformações do sistema mundial ${ }^{3-6}$. Há, entretanto, alguns aspectos gerais que podem ser enumerados sem pretensão de aprofundamento interpretativo, objetivando delinear as transformações mais evidentes do panorama global nessa transição de milênio: a derrocada do projeto socialista soviético; as repetidas crises econômicas do bloco capitalista; a emergência de países antes incluídos na categoria de subdesenvolvidos e que passaram a ser designados de renda intermediária ou países emergentes; consolidação da influência em escala global da China; e, sombreando os aspectos anteriores, a persistência dos Estados Unidos como centro mundial de poder. Nesse contexto ressalta um aspecto fundamental para a presente discussão, o fortalecimento dos interesses entre os países em desenvolvimento majoritariamente situados no hemisfério sul e, no bojo desse processo, iniciativas alcunhadas de cooperação sul-sul ${ }^{7}$.

A área de saúde acompanha essa evolução histórica dos processos de cooperação entre países que aspiram superar as condições de desigualdade e dependência e suas consequências la- mentáveis no plano das condições de vida e saúde das suas populações ${ }^{8}$. Paralelamente, neste início de século, a bioética ampliou sua agenda de reflexão e trabalho, até então assentada preferencialmente nos campos biomédico e biotecnológico, passando a incorporar temas das áreas sanitárias, sociais e ambientais que não faziam parte de suas preocupações centrais. Nesse sentido, o presente estudo se propõe analisar a cooperação internacional em saúde a partir da perspectiva bioética, particularmente na travessia para o novo paradigma de horizontalidade almejado no contexto da cooperação sul-sul, que pretende substituir o modelo da assistência técnica vertical patognomônico das relações norte-sul.

\section{Fundamentação teórica}

A situação mundial na área da saúde apresenta, mesmo nos países ricos, problemas relacionados ao acesso de segmentos da população ao consumo básico de bens e serviços, além de deficiências na qualidade e efetividade das ações dos sistemas de saúde. Nos países pobres, a essas dificuldades agregam-se outras, igualmente históricas, resultantes da precariedade da infraestrutura em todos os setores sociais, bem como da situação de carência econômica da maioria de suas populações. Em todos os países, tornam-se cada dia mais complexas as implicações das inovações tecnológicas sobre os serviços de saúde. Também ressaltam, com diferentes gradações, os efeitos da transição demográfica e epidemiológica, bem como as transformações impostas ao meio ambiente e que impactam as condições de saúde das pessoas e das coletividades. Subjacentes a essa problemática, desponta um aspecto indispensável para o debate, que remete para os compromissos do poder público perante a saúde como direito humano.

Esse cenário e suas projeções para o futuro têm sido objeto de estudos que revelam o paradoxal agravamento das condições de vida e saúde vis-à-vis o desenvolvimento econômico e técnico-científico. As avaliações indicam não apenas as discrepâncias entre os níveis de bem estar e riqueza nas diferentes partes do mundo e as condições sanitárias da maioria das populações, mas levantam discussões sobre os aspectos éticos envolvidos na gênese e na escolha de alternativas para enfrentamento desses problemas ${ }^{9,10}$.

A preocupação com as dimensões éticas em todos os ramos da atividade humana vem de longa data. Neste sentido, é necessário adotar “... o caminho dos valores éticos e humanos para 
orientar todo o empreendimento..."11 derivado das revoluções científicas que ocorreram no século XX. Entretanto, ao longo desse período a ética circunscreveu-se preferencialmente a regras de conduta profissional - os códigos deontológicos - desfocando a saúde como qualidade de vida, como direito humano fundamental e como processo relacional do homem no seu contexto social e com a natureza. A retomada desse ponto de vista holístico sob a designação de bioética ocorreu "quando Potter cunhou o neologismo, em 1970, nos Estados Unidos"12.

Polêmicas internacionais em torno da regulação de pesquisas envolvendo seres humanos levaram a Associação Médica Mundial a aprovar em 1964 a Declaração de Helsinki, documento referencial não apenas para as entidades nacionais vinculadas àquela instituição, pois adquiriu forte legitimidade como demonstra a história de sua aprovação e das modificações que lhe foram apostas subsequentemente. O reflexo desse embate no âmbito das Nações Unidas ocorreu apenas dois anos depois, com a proibição de “... submeter uma pessoa, sem seu livre consentimento, a experiências médicas ou científicas" constante no artigo $7^{\circ}$ do Pacto sobre Direitos Civis e Políticos das Nações Unidas, promulgado pelo Decreto $\mathrm{N}^{\circ} 592$ de 6 de julho de $1992^{13}$, que transcreve integralmente o texto original aprovado na XXI Sessão da Assembléia-Geral das Nações Unidas, em 16 de dezembro de 1966. Validado no Brasil vinte e dois anos depois, sua aplicação jurídica ainda não foi incorporada em vários países.

A Declaração Universal sobre Bioética e Direitos Humanos ${ }^{14}$, aprovada por aclamação na 33a Sessão da Conferência Geral da UNESCO em 19 de outubro de 2005 e homologada por 191 países em Assembléia Geral das Nações Unidas, proclama no Artigo 24, sob o título de "Cooperação Internacional":

a) Os Estados devem promover a disseminação internacional da informação científica e estimular a livre circulação e o compartilhamento científico e tecnológico.

b) Ao abrigo da cooperação internacional, os Estados devem promover a cooperação cultural e científica e estabelecer acordos bilaterais e multilaterais que possibilitem aos países em desenvolvimento construir capacidade de participação na geração e compartilhamento do conhecimento científico, do know-how relacionado e dos benefícios decorrentes.

c) Os Estados devem respeitar e promover a solidariedade entre Estados, bem como entre indivíduos, famílias, grupos e comunidades, com atenção especial para aqueles tornados vulnerá- veis por doença ou incapacidades ou por outras condições individuais, sociais ou ambientais e aqueles indivíduos com maior limitação de recursos.

A interpretação desse texto é irrefutável quanto à responsabilidade dos Estados na cooperação internacional voltada para o compartilhamento solidário do desenvolvimento técnico e científico e seus benefícios em termos de riqueza e bem estar. É interessante notar que as implicações bioéticas das relações internacionais ressurjam no âmbito da UNESCO na transição para o novo século, no bojo de um movimento mundial em torno dos direitos humanos. Trata-se da revalorização de compromissos já evocados em meados do século passado na Constituição da Organização Mundial da Saúde (OMS), aprovada pelas Nações Unidas em 1947, cujo preâmbulo lista nove princípios, o segundo dos quais se refere à saúde como direito humano fundamental; e os três seguintes remetem para compromissos a serem assumidos pelos Estados “... com o fim de cooperar entre si e com os outros para promover e proteger a saúde de todos os povos...”. A meio caminho dessa linha do tempo encontra-se evidente alusão no mesmo sentido na Declaração de Alma-Ata, aprovada em 1978 na Conferência Internacional sobre Cuidados Primários de Saúde promovida pela OMS e UNICEF em Alma-Ata, URSS, que propugnou a “... redução da lacuna existente entre o estado de saúde dos países em desenvolvimento e o dos desenvolvidos" e a implantação da atenção primária à saúde “... num espírito de cooperação técnica e em consonância com a nova ordem econômica internacional".

Além dos aspectos acima, as controvérsias sobre o genoma humano, patentes de produtos de consumo humano, degradação ambiental e outros temas relacionados ao porvir das condições de vida e saúde na Terra suscitam preocupações relacionadas aos desequilíbrios de poder econômico e científico que, em boa medida, engendram as condições de desigualdade e injustiças que acossam os países mais pobres. Nesse sentido, a Declaração aprovada por 191 países que participaram da Cúpula do Milênio das $\mathrm{Na}$ ções Unidas em setembro de 2000 na cidade de Nova Iorque, ao expressar os compromissos de todos os estados membros em defesa da vida humana, também respalda a perspectiva bioética como um importante modulador da cooperação internacional em saúde.

Essas considerações demonstram a relevância da bioética ante a inquietação em torno da situação e tendências da saúde mundial e seus 
desdobramentos práticos, bem como o envolvimento dos organismos internacionais com essa temática.

\section{Método}

O presente estudo sustentou-se em uma revisão bibliográfica que buscou identificar a literatura disponível na intersecção temática entre a bioética e a saúde pública no contexto da diplomacia, sem a intenção de aprofundar o estudo interpretativo das publicações resgatadas nas fontes consultadas, além de restringir essa busca a artigos divulgados em periódicos científicos. Ou seja, o propósito da revisão limitou-se a fazer uma primeira aproximação à produção científica demonstrativa da possível interação entre estes três campos que se pretende estudar.

A busca da literatura foi direcionada aos sistemas bibliográficos virtuais de duas bibliotecas de referência internacional: a Biblioteca Virtual em Saúde, do Centro Latino-Americano e do Caribe de Informação em Ciências da Saúde (BVS/Bireme), órgão especializado da OPAS/ OMS mantido com apoio do governo brasileiro e sediado em São Paulo; e a PubMed, um acervo na Internet organizado e mantido pelo Centro Nacional de Informação em Biotecnologia, da Biblioteca Nacional de Medicina, vinculada ao Instituto Nacional de Saúde dos Estados Unidos.

As consultas foram orientadas pela seguinte metodologia: definição de "termos de busca" (especificados nas tabelas de dados adiante apresentadas) e utilização dos mesmos na execução de rotinas de recuperação próprias dos sistemas bibliográficos. As listagens obtidas com essa metodologia foram utilizadas para mapear a abordagem interdisciplinar entre os campos de conhecimento em tela, além de fornecerem subsídios bibliográficos para o estudo. A análise dessa literatura teve um caráter exploratório, mediante leitura do título e resumo dos artigos, capturando impressões que orientassem sua localização na zona fronteiriça dos campos de conhecimento referidos.

\section{Resultados e Discussão}

Uma observação inicial é que não há consenso sobre os descritores adotados pelas diversas fontes bibliográficas. Como se vê na Tabela 1, a BVS/ Bireme cataloga 424 artigos publicados em revistas científicas sobre cooperação técnica entre países, praticamente não apresentando resultados para a busca com os termos diplomacia da saúde e cooperação sul-sul. Já a PubMed lista 114 artigos sobre diplomacia da saúde (health diplomacy), um montante vinte vezes maior sobre cooperação sul-sul (south-south cooperation) e 589 artigos sobre cooperação técnica entre países (technical cooperation among countries).

Os dados revelam uma participação diminuta do temário em foco (linhas á, $\beta$ e $\gamma$ na Tabela 1 ) no conjunto da produção científica em ciências da saúde, mesmo considerando somente a literatura catalogada sobre cooperação internacional e saúde pública (linhas ä e å), onde seguramente se incluem os três conjuntos anteriores. Além disso, há superposição entre as citações daquelas duas bibliotecas, pelo que não se pode adotar a soma de seus acervos como o total da literatura disponível sobre o temário em consideração.

A articulação entre saúde e diplomacia é tema frequente em publicações de instituições oficiais nacionais e agências das Nações Unidas, particularmente a OPAS/OMS. O que não se verifica na

Tabela 1. Número de artigos nas bases de dados BVS/BIREME e PubMed/NCBI, segundo diferentes termos de busca.

\begin{tabular}{lcc}
\hline \multicolumn{1}{c}{ Termos de busca } & BVS $^{\mathbf{a}}$ & PubMed $^{\mathbf{b}}$ \\
\hline "diplomacia da saúde" (á) & $1(3)$ & $8(114)$ \\
"cooperação sul-sul" $(\beta)$ & - & $238(2.228)$ \\
"cooperação técnica entre países" $(\gamma)$ & $424(4.869)$ & $73(589)$ \\
"cooperação internacional” (ä) & $34.791(40.390)$ & $9.507(111.714)$ \\
"saúde pública" (å) & $137.528(169.132)$ & $397.737(4.756 .105)$
\end{tabular}

Fonte: BVS/BIREME; PubMed/NCBI, Brasil, 2011 [acesso eletrônico]. ${ }^{\text {a }}$ Entre parêntesis: número total de referências recuperadas a partir do termo de busca (além de artigos, inclui documentos das Nações Unidas, monografias, dissertações, teses e recursos educacionais abertos). ${ }^{\mathrm{b}}$ Entre parêntesis: número total de artigos citados recuperados a partir do termo de busca, incluídos os artigos revisados (review). 
literatura acadêmica, conforme sugere o pequeno número de artigos sobre cooperação técnica entre países na BVS/Bireme: apenas 424 (8,7\%) desse tipo de referências dentre o total de 4.869 citações, onde predominam documentos de organismos intergovernamentais ou nacionais. Esses dados são indícios da aproximação entre os operadores das políticas governamentais de saúde e diplomacia, convívio que ainda não se espraiou no ambiente acadêmico, onde rareiam análises com esse enfoque e são recentes os relatos de programas de ensino e pesquisa nesses dois campos de conhecimento ${ }^{15-18}$.

Um resultado significativo desse estudo bibliográfico, a partir da análise exploratória do conteúdo dos artigos científicos, é que raramente os autores focalizam as questões de saúde estabelecendo correlações ou remetendo ao debate entre políticas de saúde e relações externas dos países ${ }^{19}$. A maioria refere-se a aspectos específicos de saúde pública, predominantemente nas áreas de epidemiologia ou administração sanitária, eventualmente cotejando situações ou indicadores de saúde em diferentes contextos nacionais.

Contudo, são trabalhos que apontam as dificuldades enfrentadas pelos países e assinalam demandas ou oportunidades de cooperação enquanto estratégia vantajosa para fortalecimento dos respectivos sistemas de saúde, aproveitando as capacidades e os potenciais disponíveis nos diversos contextos nacionais. Discussões e propostas nesse sentido povoam a agenda da OPAS/ OMS há várias décadas, como bem ilustra uma publicação ${ }^{20}$ composta de vinte artigos e seis estudos de caso de países, incluindo uma discussão sobre a cooperação internacional nas reformas setoriais nas Américas. A valorização das relações entre saúde e política exterior nessa região culminou com a aprovação de uma resolução do Conselho Diretor da OPAS ${ }^{21}$ em outubro de 2008, que traça orientações e recomendações a serem seguidas por essa agência intergovernamental, bem como pelos próprios países.

As conexões entre saúde e diplomacia são históricas $^{22-23}$, embora se contem poucos estudos na interseção desses temas no Brasil ${ }^{24-25}$, a despeito do expressivo protagonismo de sanitaristas brasileiros no cenário da diplomacia, como ocorreu nas tratativas para inclusão da saúde na Carta das Nações Unidas e a subsequente criação da Organização Mundial da Saúde ${ }^{26}$. A revisão acima apontada delineia as características da bibliografia sobre a saúde no contexto das relações diplomáticas entre países, não se encontrando nessa literatura uma abordagem das contra- dições ou tensões entre essas duas perspectivas, como se indica a seguir.

\section{Diplomacia em saúde}

O ponto de partida para a análise aqui proposta é a interpretação da diplomacia em saúde como uma área de atuação política e técnica situada na fronteira entre dois campos de conhecimento independentes e de certa forma isolados. Essa significação advém do distanciamento preservado entre as culturas e as práticas institucionais da diplomacia e da saúde pública, onde os atores de ambos os lados, sanitaristas e diplomatas, manquejam numa trabalhosa e escassa convivência, sob a influência de concepções doutrinárias antagônicas, o realismo da diplomacia e o altruísmo da saúde. Como é da natureza das fronteiras, a delimitação entre diplomacia e saúde pública, além de um lugar de conexões é também um ambiente de conflitos, onde o convívio é tensionado entre os interesses próprios dos países vis-à-vis os postulados virtuosos da saúde pública.

Para onde apontaria a resultante desse encontro ou confronto como vetor guia da cooperação internacional em saúde? Na direção de equidade e justiça ou na vertente do exercício de um poder suave da diplomacia (soft power), capaz de influenciar outros povos para obter resultados desejados sem usar mecanismos de coerção econômica, militar ou de outra índole ${ }^{27}$ ? A diplomacia da saúde pode ser fiel aos ideais de equidade e justiça e, por conseguinte, a cooperação técnica nessa área representa um eixo de articulação entre as políticas de saúde e de relações exteriores em prol dos direitos humanos na área de saúde? Ou, por outro lado, constitui apenas um instrumento útil a serviço de interesses econômicos (controle sanitário de produtos comerciais), de segurança nacional (controle de circulação e difusão de doenças)? Enfim, seria apenas um ardil para atenuar a crueza das relações internacionais no marco das disputas de poder entre as nações?

Insinuar essas indagações não nega o caráter dialético entre o ser e dever ser das relações entre saúde e diplomacia, nem tampouco pressupõe necessariamente maior hierarquia à política de relações intenacionais no bojo da qual se institui a diplomacia da saúde. Em ambos os casos, tanto sob o domínio das boas intenções como dos interesses frequentemente contrapostos, é válido insistir no questionamento acima no sentido de mapear, prevenir e combater desafios bioéticos resultantes ou implícitos nas políticas e nos pro- 
gramas de cooperação técnica constituintes da diplomacia da saúde.

Em resumo, ao vislumbrar relações entre diplomacia e saúde pública ${ }^{28}$ é indispensável levar em conta as contradições presentes nos contextos nacionais e internacionais, suas conformações estruturais, bem como o caráter mutante das circunstâncias históricas. Instigar o diálogo sobre a inserção da saúde na política diplomática reforça a convicção sobre a relevância da bioética no trato das contradições aí subjacentes, mote deste ensaio.

\section{Bioética e diplomacia em saúde}

As fontes bibliográficas referidas anteriormente disponibilizam elevado número de artigos sobre bioética no conjunto da literatura sobre ciências da saúde, mas apresentam resultados menores quando as consultas buscam a interseção com outros temas, conforme exposto na Tabela 2.

Embora os levantamentos bibliográficos anteriores (Tabela 1) tenham recuperado citações na interseção diplomacia e saúde, cooperação sulsul e cooperação técnica entre países, ao cruzar esses temas com bioética os resultados foram nulos em ambas as bibliotecas. As consultas foram mais animadoras ao acasalar bioética com o termo genérico de cooperação internacional; mas, ao tentar uma apuração mais refinada, acrescentando saúde pública ao binômio anterior, os resultados foram mais escassos. Por fim, ao adotar a alternativa de pesquisa com termos correlatos a cooperação internacional (relações internacionais, saúde internacional, saúde global), a soma desses resultados triplicou a recuperação bibliográfica anterior.
Os resultados dessa estratégia de recuperação bibliográfica sugerem expressiva produção científica onde garimpar a temática de cunho interdisciplinar entre bioética e diplomacia em saúde: coleções de 93 artigos citados na BVS/Bireme e 161 artigos revisados (review) na PubMed (última linha da Tabela 2), mesmo considerando a salvaguarda assinalada anteriormente sobre duplicatas entre essas listas de referências. Contudo, os dados preliminares sobre os conteúdos dessas publicações indicam frágil abordagem interdisciplinar, com ênfase ora sobre os aspectos da bioética ora da saúde pública, ou mesmo explorando as articulações entre esses dois temas, sem incursionar pelas dimensões de cooperação no contexto das relações ou acordos diplomáticos entre países.

O temário inclui outros campos de conhecimento além da bioética, saúde pública e diplomacia, como a história, a economia, a sociologia e a antropologia. Sem desconhecer essas possibilidades, mas apenas visando manter a delimitação do escopo do presente estudo, os comentários seguintes remetem exclusivamente à perspectiva bioética ante a cooperação internacional em saúde. Essa anotação alerta para a limitação do recorte temático adotado nesta análise exploratória, reconhecendo a necessidade de ampliação da referida abordagem de modo a proporcionar uma aproximação mais apropriada ao objeto de estudo.

Além da restrição acima, os comentários finais dessa apreciação interdisciplinar limitam-se ao escopo da bioética desenvolvida na América Latina a partir das formulações propostas pela Cátedra UNESCO de Bioética da Universidade de Brasília, sob a designação de Bioética de Intervenção, abordagem que ampliou sua esfera de

Tabela 2. Número de artigos nas bases de dados BVS/BIREME e PubMed/CNBI, segundo diferentes termos de busca.

\begin{tabular}{lrr}
\hline \multicolumn{1}{c}{ Termos de busca } & BVS/BIREME & PubMed $^{\text {a }}$ \\
\hline $\begin{array}{l}\text { "bioética" } \\
\text { "bioética and saúde pública" }\end{array}$ & 8.424 & $19.385(1.976)$ \\
"bioética and diplomacia da saúde" & 348 & $6.759(693)$ \\
"bioética and cooperação sul-sul” & - & - \\
"bioética and cooperação técnica entre países" & - & - \\
"bioética and cooperação internacional” & 2 & - \\
"bioética and saúde pública and cooperação internacional” & 304 & $925(74)$ \\
"bioética and saúde pública and ...” & 33 & $444(42)$ \\
\hline
\end{tabular}

Fonte: BVS/BIREME; PubMed/NCBI, Brasil, 2011 [acesso eletrônico]. a Entre parêntesis: número de artigos revisados (review) dentre o total de artigos citados. ${ }^{\text {b }}$ Soma de quatro variações do termo de busca, com os seguintes finais: (1) cooperação internacional, (2) relações internacionais, (3) saúde internacional e (4) saúde global. 
estudos e investigações para o campo das políticas públicas ${ }^{29}$.

O marco histórico desse enfoque foi datado com os avanços doutrinários consagrados no Sixth World Congress of Bioethics, realizado em Brasília em 2002. Alguns anos depois, a Declaração Universal sobre Bioética e Direitos Humanos conferiu reconhecimento institucional no âmbito das Nações Unidas para essas mudanças paradigmáticas no conceito da bioética ${ }^{30}$, abrindo horizontes para novas formas de intervenção do poder público ${ }^{31}$.

Vários itens da referida Declaração tratam dos aspectos bioéticos nos processos de cooperação internacional bilateral ou multilateral, que se aplicam às políticas e planos governamentais envolvendo os setores de saúde de dois ou mais países, seja no seu preâmbulo ou no próprio corpo da mesma. Ao estudar a participação do Brasil na elaboração e aprovação dessa Declaração, Barbosa $^{32}$ analisa o compromisso do país com seus princípios e recomendações, aspectos já prenunciados em uma "radiografia bioética do Brasil” realizada alguns anos antes ${ }^{33}$.

A discussão sobre a inclusão social na epistemologia da bioética ${ }^{34}$ é uma senda a ser explorada e aprofundada para analisar as dimensões bioéticas da cooperação internacional em saúde, principalmente quando se trata de países marcados por acentuado grau de exclusão e pobreza. Nessa linha de interpretação se inclui a abordagem da Bioética de Intervenção para países periféricos no contexto de "poder e injustiça"29, explicitada claramente nas seguintes palavras:

Dadas as gritantes desigualdades entre os países centrais e periféricos nas condições de acesso aos bens e serviços que caracterizam a qualidade de vida, circunscrever a discussão ética sobre as formas de vida e as razões da morte apenas às relações estabelecidas no âmbito da prática clínica ou da pesquisa de medicamentos revela-se não apenas um eufemismo moral, mas uma cegueira ética ${ }^{35}$.

\section{Considerações finais}

Os dados levantados nesse estudo parecem demonstrar a relevância da bioética no contexto da cooperação internacional em saúde no âmbito das agências das Nações Unidas e fóruns de países, além de ressaltar a contribuição da bioética brasileira e latino-americana para esse desígnio. A incursão bibliográfica desenvolvida apurou o enfoque bioético na literatura científica mundial sobre as ciências da saúde em geral e a saúde pública em particular e, em escala mais reduzida, sobre temas da cooperação internacional nessa área. Em suma, são argumentos que abonam a ampliação de estudos e investigações e reforçam a convicção sobre a contribuição da bioética para o desenvolvimento dos processos de cooperação alicerçados em postulados de solidariedade, equidade, justiça e outros referenciais utilizados na conformação e sustentação epistemológica da disciplina.

No plano prático e aplicado, a sistematização e o aprofundamento do conhecimento sobre o objeto de reflexão aqui proposto visa proporcionar subsídios para o equacionamento de alguns dos desafios que afrontam o sistema de cooperação internacional, cujas expectativas estão situadas não somente no plano do conhecimento, mas também na seara da política e da prática institucional.

No plano teórico, trata-se de aportar elementos em torno da questão se a diplomacia em saúde é uma área de atuação política e técnica que tenderia a constituir-se em campo de conhecimento específico, ou permanecer num limbo interdisciplinar que atualmente reúne diversas denominações como saúde internacional ou saúde global, efluentes mal acomodados no leito da saúde pública tradicional. Essa conjectura pode se beneficiar da acepção sobre campo científico formulada por Bourdieu ${ }^{36}$, numa contribuição relevante para a construção de um conhecimento transdisciplinar envolvendo diversas áreas ou campos do saber, a partir do encontro da bioética de intervenção com a saúde pública ${ }^{12}$ em miscigenação virtuosa com as ciências sociais, aqui destacada a indicação das relações internacionais, ou mais especificamente, da diplomacia.

No campo das políticas institucionais de cooperação internacional em saúde, a contribuição será certamente diminuta, ante a magnitude e a complexidade dos problemas dessa agenda. Mas a intenção otimista alimenta a crença de que toda ajuda, por modesta que possa ser, é bem vinda quando se almeja realinhar os desequilíbrios de poder político, econômico, técnico e científico de modo a encurtar ao invés de alargar as diferenças entre as condições de vida e saúde entre países centrais e periféricos.

Este estudo procurou acenar para a projeção da bioética no cenário da diplomacia em saúde ao apostar nessa perspectiva como referencial de análise da cooperação internacional em saúde, ou seja, como pauta para esses processos nos marcos da solidariedade, equidade e justiça, de forma a contribuir para a redução das desigualdades nas condições de vida e saúde entre as nações. O corolário 
dessa apreciação se aplica de forma mais ajustada às experiências ou projetos de cooperação sul-sul, dado o ideário subjacente a esse modelo de relações, contemplando postulados de horizontalidade e consenso, solidariedade e altruísmo, equidade e justiça no relacionamento entre países.

$\mathrm{O}$ enfoque aqui desenvolvido não pretende reduzir o conceito de diplomacia em saúde ao significado da cooperação sul-sul, ou mesmo ao sentido mais amplo de cooperação internacional em saúde. De igual maneira, não restringe o campo de ação da bioética, que obviamente abarca o conjunto das relações internacionais, expandindo-se para além da saúde ou dos processos de cooperação nessa área, ou especificamente da cooperação sul-sul em saúde. A intenção é apenas realçar as acepções sobre ambos os termos que pontuam a argumentação aqui exposta: de um lado, a vertente "periférica e anti-hegemônica” da bioética de intervenção, que vicejou no Brasil com profundas ligações com a saúde coletiva $^{12}$; de outro, a expressão da diplomacia em saúde concretizada na cooperação sul-sul em saúde, nos moldes da experiência brasileira ${ }^{8,37}$.

\section{Colaboradores}

JP Santana e V Garrafa participaram igualmente de todas as etapas de elaboração do artigo. 


\section{Referências}

1. Amador EA. El Nuevo rostro de la cooperación técnica entre países en desarrollo (CTPD) y las nuevas tendencias internacionales. Rev. Ciencias Sociales 2001; 1(94):169-188.

2. Ha-Joon C. Maus samaritanos: o mito do livre comércio e a história secreta do capitalismo. Rio de Janeiro: Elsevier; 2009.

3. Landes DS. A riqueza e a pobreza das nações: por que algumas são tão ricas e outras tão pobres. Rio de Janeiro: Campus; 1998.

4. Guimarães SP. Desafios brasileiros na era dos gigantes. Rio de Janeiro: Contraponto; 2005.

5. Hobsbaum EJ. Era dos extremos: o breve século XX: 1919-1991. São Paulo: Companhia das Letras; 1995.

6. Fiori JLF. Prefácio ao poder global. Rev. Tempo do Mundo 2010; 2(1).

7. Boron AA, Lechini G. Política y movimientos sociales en un mundo hegemónico. Lecciones desde África, Asia y AméricaLatina [monografia]. Buenos Aires: Consejo Latinoamericano de Ciencias Sociales; 2006.

8. Santana JP. Um olhar sobre a Cooperação Sul-Sul em Saúde. Cien Saude Colet 2011; 16(6):2415-2424.

9. Garrafa V, Oselka G, Diniz D. Saúde pública, bioética e equidade. Bioética 1997; 5(1):27-33.

10. Benatar SR, Daar AS, Singer PA. Global health challenges: the need for an expanded discourse on bioethics. PLoS Med 2005; 2(7):e143.

11. Pessini L, Hossne WS. Revoluções científicas e necessidade de ética. Bioethikós 2011; 5(1):7-9.

12. Porto D, Garrafa V. A influência da Reforma Sanitária na construção das bioéticas brasileiras. Ciênc. Saúde Coletiva 2011; 16(1):719-729.

13. Brasil. Decreto $N^{\circ} 592$ de 6 de julho de 1992. Pacto internacional sobre direitos civis e políticos. Diário Oficial da União 1992; jul 7.

14. Cátedra UNESCO de Bioética da Universidade de Brasília; Sociedade Brasileira de Bioética. Declaração Universal sobre Bioética e Direitos Humanos. Brasília: 2005.

15. Almeida C. A experiência da Fiocruz na formação de profissionais em saúde global e diplomacia da saúde: base conceitual, estrutura curricular e primeiros resultados. R. Eletr. de Com. Inf. Inov. Saúde 2010; 4(1):148-164.

16. Novotny T; Adams V. Global Health Diplomacy. Global Health Sciences Working Paper 2007: 1-10.

17. Kickbusch I, Novotny TE, Drager N, Silberschmidt G, Alcazar S. Global health diplomacy: training across disciplines. Bull World Health Organ 2007; 85(12):971-973.

18. Kickbusch I. Global health diplomacy: how foreign policy can influence health. BMJ 2011; 342(d3):154.

19. Roa AC, Santana JP. Cooperação Sul-Sul em Saúde nos Contextos Sub-Regionais da América Latina. In: $3^{\circ}$ Encontro Nacional da ABRI; 2011; São Paulo, Sp; 2011

20. Revista Panamericana de Salud Pública [periódicos na internet]. 2000; 8(1-2). [acessado 2011 ago 18]. Disponível em: http://www.scielosp.org/scielo.php? script $=$ sci_issuetoc\&pid $=1020-498920000007 \& \operatorname{lng}$ $=\mathrm{pt} \& \mathrm{nrm}=\mathrm{iso}$
21. $48^{\circ}$ Conselho Diretor, 60 a Sessão do Comitê Regional. Resolução CD48. R16. A saúde e as relações internacionais: seu vínculo com a gestão do desenvolvimento nacional da saúde. [acessado 2011 ago 18]. Disponível em: http://www.paho.org/portuguese/ gov/cd/CD48.r16-p.pdf

22. Fidler DP. The globalization of the public health: the first 100 years of international health diplomacy. Bull World Health Organ 2001; 79(9):842-849.

23. Lima NT. O Brasil e a Organização Pan-Americana da Saúde: uma história de três dimensões. In: Finkelman J, organizador. Caminhos da Saúde Pública no Brasil. Rio de Janeiro: Fiocruz; 2002.

24. Alcazar S. A inserção da saúde na política exterior brasileira. In: $48^{\circ}$ Curso de Altos Estudos do Instituto Rio Branco. Brasília: Ministério das Relações Exteriores; 2005.

25. Rubarth EO. A diplomacia brasileira e os temas sociais: o caso da saúde. Brasília: Instituto Rio Branco; 1999.

26. Sze S. WHO: from small beginnings [interview]. World Health Forum 1988; 9(1):29-34

27. Nye Júnior JS. Public Diplomacy and Soft Power. The Annals of The American Academy of Political and Social Science 2008; 616(1):94-109.

28. Fidler DP. Reflections on the revolution in health and foreign policy. Bull World Health Organ 2007; 85(3):243-244.

29. Garrafa V, Porto D. Intervention bioethics: a proposal for peripheral countries in a context of Power and injustice. Bioethics 2003; 17(5-6):399-416.

30. Saada A. La Declaración Universal sobre Bioética y Derechos Humanos: ampliación democrática para una sociedad mas justa. Rev. Brasileira Bioética 2006; 2(4):413-422.

31. Garrafa V. Verbete: Ética y política. In: Tealdi JC, organizador. Diccionario Latinoamericano de Bioética. Bogotá: Unibiblos/Redbioética Unesco; 2008. p. 531-533.

32. Barbosa SN. Bioética no Estado brasileiro: situação atual e perspectivas futuras. Brasília: Universidade de Brasília; 2010.

33. Garrafa V. Radiografia bioética de um país - Brasil. Acta Bioethica 2000; 6(1):171-175.

34. Garrafa V. Inclusão social no contexto político da bioética. Rev. Brasileira Bioética 2005; 1(2):122-132.

35. Porto D, Garrafa, V. Bioética de Intervenção: considerações sobre a economia de mercado. Bioética (CFM) 2005; 13(1):111-123.

36. Bourdieu P. Os usos sociais da ciência: por uma sociologia clínica do campo científico. São Paulo: UNESP; 2003.

37. Buss P, Ferreira JR. Diplomacia da saúde e cooperação Sul-Sul: as experiências da Unasul saúde e do plano estratégico de cooperação em saúde da Comunidade de Países de Língua Portuguesa (CPLP). R. Eletr. de Com. Inf. Inov. Saúde 2010; 4(1):106-118

Apresentado em 20/08/2011

Aprovado em 24/02/2012

Versão final apresentada em 01/03/2012 